\title{
SINGULAR VALUE DECOMPOSITION VS DUALITY APPROACH IN AHP: TIME AND RANK COMPARISONS IN FACULTY MEMBER SELECTION
}

\author{
Nur Jumaadzan Zaleha Mamat, Jacob K. Daniel \\ Faculty of Information Technology, \\ Multimedia University, \\ 63100 Cyberjaya, \\ Selangor Darul Ehsan, \\ Malaysia. \\ zaleha@mmu.edu.my,jacob.daniel@mmu.edu.my
}

Keywords: singular value decomposition (SVD), duality approach, Analytic Hierarchy Process (AHP), time and rank comparisons, faculty member selection

\begin{abstract}
Summary: Using Saaty's original AHP, faculty member selection committee faces great challenges when the number of candidates is large. Two methods, singular value decomposition (SVD) in AHP and the duality approach to reduce the number of pairwise comparisons are studied and compared in terms of time complexity and overall rank result. Experiments showed that the SVD is preferred over the duality in AHP.
\end{abstract}

\section{Introduction}

The Analytic Hierarchy Process (AHP) is a multi-criteria decision making method, first introduced by T.L. Saaty (Saaty, 1980 and 1994) in the 70s. The principles of the AHP are logic, comprehensive, and it can be used in both quantitative and qualitative multi-criteria decision making problems.

In the AHP, the multi-criteria decision making problem is structured into a hierarchy. At the top of the hierarchy, which is called the first level, the main objective that the decision maker is aiming will be depicted. In order to ease the decision process, the problem is broken down into sections based on all possible related criteria that contribute to the decision making. These selection criteria form the second level of the hierarchy. The decision maker may even decompose the problem further by considering subcriteria under each one of the selection criteria in level two. This exercise is believed to further assist the decision maker in achieving the best decision. The size of the hierarchy depends on the complexity of the problem and also the discretion of the decision maker. Lastly, all decision alternatives are put at the lowest level of the hierarchy.

Once the problem is hierarchically structured, the decision maker would need to compare decision alternatives, two at a time under each one of the selection criteria or sub-criteria. This is called pairwise comparisons. All the comparisons are put into square matrices. These pairwise comparisons are needed to determine the priorities/weights of the alternatives with respect to the individual selection criterion that will later be used to obtain the priorities/weights of those alternatives in terms of the overall objective. If the priorities/weights of the selection criteria were not predetermined, then the decision maker will have to make pairwise comparisons of the selection criteria. As the AHP is also suitable for qualitative problems, Saaty's scale of relative importance is used in order to obtain the pairwise comparisons.

The last principle of the AHP is to synthesize the priorities/weights of all elements in the hierarchy through the matrices of pairwise comparisons as mentioned above. Saaty suggested the Eigenvector Method (EM). In the EM, it is the right principle eigenvectors of the pairwise comparison matrices that are estimated. Take one pairwise comparison matrix say, pairwise comparisons of decision alternatives 
under criterion A. First, the sum for each column in the matrix is calculated, and then all elements in the column are divided by that column's sum. This will results in a new normalized matrix. Next, the values of all elements in each row of the new normalized matrix are added. Then the row sum is divided by the number of elements in the row. The results would be the priorities/weights of the decision alternatives under selection criterion A. Do the same for all pairwise comparison matrices.

Last is to calculate the overall ranking or priorities/weights of decision alternatives. Put all weight vectors of decision alternatives under each of the selection criteria into a matrix. Then, multiply this matrix with the weight vector of the selection criteria. Hence, the decision maker will now be able to acquire the best decision for his/her problem.

To ensure that the decisions made from the results of the AHP are acceptable, due to human ability in making comparisons, the pairwise comparisons given by the decision maker need to be consistent. Thus the consistency ratio (C.R.) is set to be less than or in the neighbourhood of $10 \%$. If not, the decision maker needs to re-evaluate or re-judge his/her preferences. Interested readers on the formulation can refer to Saaty, 1980 and 1994. However, authors used own calculated Random Index (R.I) in the calculation of the C.R. for this study.

Like any other multi-criteria decision making methods, the AHP has been studied and criticized. Those include Johnson, Beine and Wang, 1979; Belton and Gear, 1983; Dyer and Wendell, 1983; Donegan, Dodd and McMaster, (1992); Triantaphyllou and Mann, 1994, M. T. Escobar and J. M. MorenoJiménezto (2000), to name a few. Much to the criticism, the AHP is one of the most accepted and widely used methods by academician as well as decision makers in various applications, especially with the birth of Expert Choice Software in 1990. Researchers and academician have also combined the AHP with other methods such as fuzzy logic (Yager, 1978; Laarhoven and Pedrycz, 1983; Boender, de Graan and Lootsma, 1989), linear programming (Ramanathan and Ganesh, 1995; Ghodsypur and O’Brien, (1998)), goal programming (Radasch and Kwak (1998); Badri (2001)) and so on.

As flexible and comprehensive as it is, decision makers do face great challenges and difficulties in having consistent pairwise comparisons of all elements in a problem, especially when there are a large number of decision alternatives, hence the time complexity and justification of the overall rankings issues.

This paper looks at two methods in dealing with the two above-mentioned issues, i.e. the singular value decomposition (SVD) in AHP and the duality approach in AHP, in the case of faculty member selection. Section 2 looks at the structure of the two methods in faculty member selection in general. Comparison on the time complexity of the two is discussed in section 3 while section 4 takes on the comparison on the overall ranking results. In the last section, conclusions and future direction of the study are given.

\section{Methods of Multi-Criteria Decision Making in Faculty Member Selection}

The case study is faculty member selection. Although some literature has been found on the staff/personnel/member selection using some models, the focus is on general human resource management aspect. Petrovic-Lazarevic (2001) presented a multi-criteria personnel selection fuzzy decision model based on the AHP for short listing and hiring decision. Iwamura and Lin (1998), Labib, Williams and O’Connor (1998) and Lai (1995) used the AHP in their study of the personnel selection process. None is specifically targeted to the selection of faculty members in a learning institution using the AHP or its variants.

The best person among a number of qualified candidates is to be decided to be the new member of a faculty. Traditionally, candidates write-in or apply online or get invited to try for a post. Interviews and/or maybe written test(s) and/or presentations are required of the candidates before the selection committee discuss among themselves on who the best candidate should be. There are times where the committee comes to a dead end, thus leading to more interviews/tests/presentations and discussions. Since this faculty member selection is a multi-criteria decision making problem, authors propose the 
usage of the AHP, but for the purpose of this paper, restrict the hierarchy to selecting an academician, with only three levels: top level - selecting the best candidate; second level - the selection criteria consisting of academic qualification, working experience, leadership qualities and some general traits; bottom level - a group of candidates eyeing for the academic post. Candidates are then compared pairwisely under each selection criterion to see whom the relatively better one is. The hierarchy is illustrated in Figure 1 below, with 6 decision alternatives. In reality, there can be more than 4 selection criteria and more than 6 candidates.

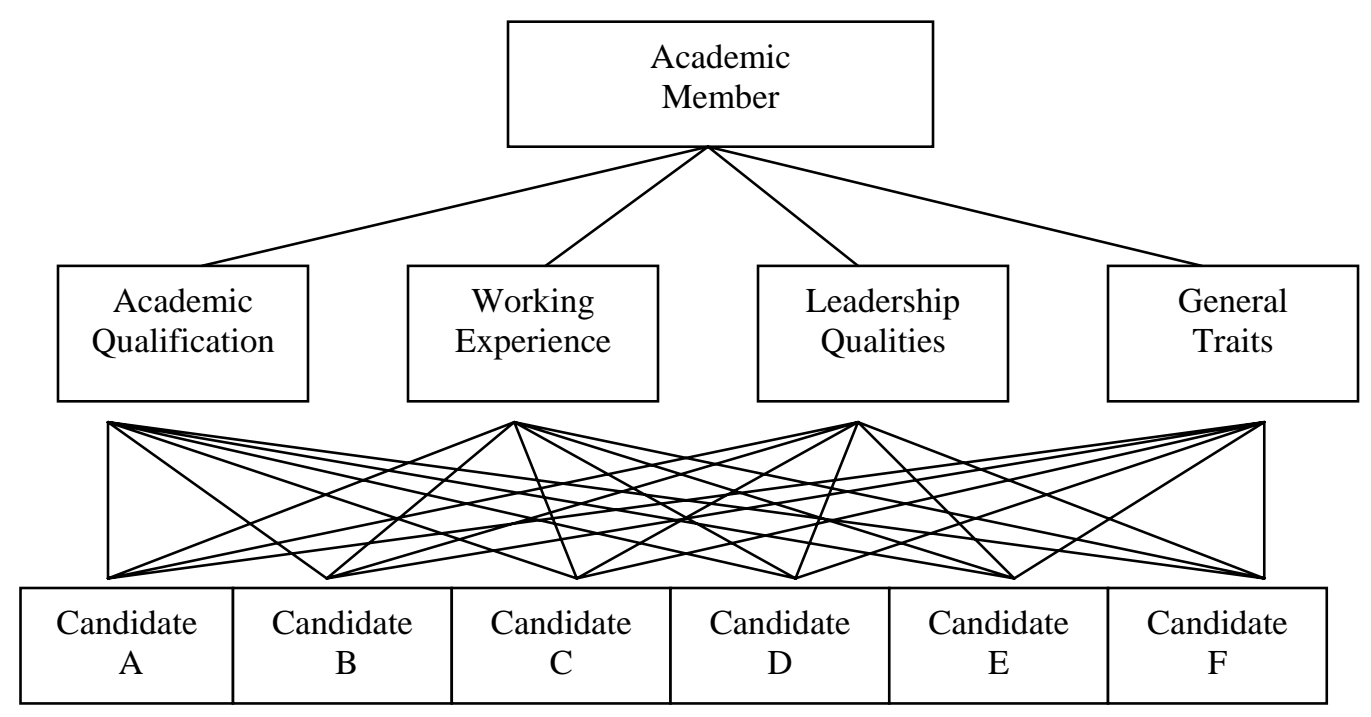

Figure 1: An AHP problem of selecting the most suitable academic member in a faculty

As mentioned in the above section, the number of candidates competing for the post can be quite large, especially when the post is advertised widely in the newspaper, radio, television and on the internet. Hence having consistent pairwise comparisons is a great challenge in the AHP in terms of time consumption and the justified overall ranking results. Therefore, authors studied the two methods, SVD and duality approach in AHP to deal with these issues. Quoting Gass and Rapcsák (2004), they proved that the rank one left and right singular vectors, that is the vectors associated with the largest singular value, yield theoretically justified weight, unlike the eigenvector method by Saaty which only looks at the right eigenvector corresponding to the maximum eigenvalue $\lambda_{\max }$ of the matrix. Triantaphyllou (1999) introduces the duality approach in order to reduce the total number of pairwise comparisons required when the number of decision alternatives is larger than the number of selection criteria plus one. Authors generated a number of random data to fit the AHP formulation, incorporate both SVD method and duality approach into it while capturing the amount of time for the two methods to get the overall rankings of the candidates.

\subsection{Singular Value Decomposition (SVD) in AHP}

The SVD is a useful and important tool in quite a wide area of studies and practicalities. To name a few, those include factor analysis, image processing, face recognition, geophysical inversion, multiple linear regression and gene expression analysis. Gass and Rapcsák (2004) incorporated the SVD and the theory of low rank approximation of a (pairwise comparison) matrix (to determine the associated weights) in the AHP for a more justified result in the decision making process.

The SVD of an $m \times n$ matrix $A$ is the decomposition of the matrix into the product of three special matrices. 
From Greenacre (1984),

Theorem 2.1.1. Any real matrix $A$ with rank $k(k \leq \min (m, n))$, can be expressed in the form of

$$
A=U D V^{T}
$$

where $U$ is an $m \times k$ matrix such that $U^{T} U=I$, $D$ is a $k \times k$ diagonal matrix with positive diagonal elements $\lambda_{1}, \lambda_{2}, \ldots, \lambda_{k}$, and $V$ is an $n \times k$ matrix such that $V^{T} V=I$. The columns of $U$ and $V$ are orthonormal in the Euclidean sense.

Another equivalent form of (1) in terms of diads is

$$
A=\sum_{i=1}^{k} \lambda_{i} u_{i} v_{i}^{T}
$$

where the $\lambda_{1}, \lambda_{2}, \ldots, \lambda_{k}$ (diagonal entries of D) are called singular values, while $u_{1}, u_{2}, \ldots, u_{k}$ and $v_{1}, v_{2}, \ldots, v_{k}$ are the columns of $\mathrm{U}$ and $\mathrm{V}$, respectively, and are termed the left and right singular vectors . If the singular values $\lambda_{k^{*}+}, \lambda_{k^{*}+2}, \ldots, \lambda_{k}$ are small when compared to $\lambda_{1}, \lambda_{2}, \ldots, \lambda_{k^{*}}$ for some $k^{*}<k$, then by taking out the last $k$ - $k^{*}$ terms of the right-hand side of the diads equation above, a good approximation of $A$ is obtained with a $k^{*}$-dimensional matrix.

The theory of low rank approximation by Eckart and Young (1936) states:

Theorem 2.1.2. Let

$$
A_{\left[k^{*}\right]}=\sum_{i=1}^{k^{*}} \lambda_{i} u_{i} v_{i}^{T}
$$

be the $m \times n$ matrix of rank $k^{*}$ formed from the largest $k^{*}$ singular values and the corresponding singular vectors of $A$. Then, $A\left[k^{*}\right]$ is the rank $k^{*}$ least squares approximation of $A$ in that it minimizes the function

$$
\|A-X\|_{F}^{2}=\sum_{i=1}^{m} \sum_{j=1}^{n}\left(a_{i j}-x_{i j}\right)^{2}
$$

for all matrices $X$ of rank $k^{*}$ or less.

Consider the SVD of pairwise comparison matrix, from Gass and Rapcsák (1998).

Theorem 2.2.3. The SVD of a positive, consistent matrix $A$ is the diad

$$
A=\frac{c_{1}}{c_{2}}\left(\begin{array}{c}
c_{2} w_{1} \\
c_{2} w_{2} \\
\vdots \\
c_{2} w_{n}
\end{array}\right)\left(\begin{array}{llll}
\frac{1}{c_{1} w_{1}} & \frac{1}{c_{1} w_{2}} & \cdots & \frac{1}{c_{1} w_{n}}
\end{array}\right), \mathbf{w}=(\text { wi }) \in R_{+}^{n},
$$

where $c_{1}$ and $c_{2}$ are positive constants such that $c_{1}^{2}=\sum_{i=1}^{n}\left(1 / w_{i}^{2}\right), c_{2}^{2}=\sum_{i=1}^{n}\left(1 / w_{i}^{2}\right)$, the right singular vector is equal to the left eigenvector multiplied by a normalizing constant, the left singular vector to the right eigenvector multiplied by a second normalizing constant, $R_{+}^{n}$ is the positive orthant, and $c_{1} / c_{2}$ is the only singular value of $A$.

When used in the AHP, all the w's are integers and belong to the interval [1,9] (according to Saaty's scale).

To obtain the weights in SVD, 
Theorem 2.2.4. Let a pairwise comparison matrix $A$ be given and let $\boldsymbol{u}$ and $\boldsymbol{v}$ be the left and right singular values belonging to he largest singular value of $A$, respectively. Then, priority of the decision maker, based on A, can be approximated by the uniquely determined, normalized positive weight vector

$$
w_{i}=\frac{u_{i}+\frac{1}{v_{i}}}{\sum_{j=1}^{n}\left(u_{j}+\frac{1}{v_{j}}\right)}, i=1, \ldots, n
$$

which is obtained by solving a distance-minimization and a measure-minimization problem with a unique solution of each.

Interested reader can refer to Gass and Rapcsák (2004) for all the proofs, justification and consistency measure based on SVD.

\subsection{Duality Approach in AHP}

In the original Saaty's AHP, the selection committee would have to ask themselves on the relative importance of candidate, say $A$ when compared to candidate $B$, say, under the Academic Qualification criterion, then another comparison under each one of the other criteria plus the comparisons with each one of the other candidates. If there is $n$ number of selection criteria and $m$ number of candidates, the selection committee would have to make $n(n-1) / 2+n(m(m-1) / 2)$ number of pairwise comparisons. Even for a small $n$ and $m(<8)$, making pairwise comparisons is quite a challenge for some decision makers.

Triantapyllou (1999) then proposed a method to reduce the number of pairwise comparisons via the duality approach governed by the assumption that in a given multi-criteria decision making problem the criteria influence the perception of the alternatives and vice-versa. Thus, instead of comparing two candidates under each selection criterion, the selection committee now considers the relative importance of criterion 1 (say academic qualification) when compared to criterion 2 (working experience, say) in terms of the candidate $A$, and so on. In other words, in a problem with $n$ number of selection criteria and $m$ number of decision alternatives, the $m$ number of dual pairwise comparison matrices (denoted by $D^{k}=$ $\left.d_{i j}^{k}, k=1,2, \ldots, m\right)$ would be the answers to the question "What is the relative importance $d_{i j}^{k}$ of criterion $C_{i}$ when it is compared with criterion $C_{j}$ in terms of alternative $A_{k}$ ?

Then, for each matrix $D^{k}$, the normalized weight vector corresponds to a normalized row of the decision matrix. This decision matrix is termed $H$ referring to each row being horizontally normalized. Let $h_{i j}$, $i=1,2, \ldots, m$ and $j=1,2, \ldots, n)$ denote the $(i, j)$ element of the decision matrix normalized in terms of each row via a sequence of $m$ dual judgment matrices with dual comparisons. Suppose also that a normal $m \times m$ pairwise comparison matrix is formed, say $O^{k}$, and it produces a vertically normalized weight vector $v_{i k}$ (for $i=1,2, \ldots, m$ ). With $h_{i j}$ values and $v_{i k}$ values, it is possible to derive the elements of any column in the decision matrix normalized in terms of each column, creating the matrix $V$ by using the formula

$$
h_{i j}^{\prime}=\left(\frac{h_{i k}}{v_{i k}}\right) v_{i j}
$$

However, when the above elements $h_{i j}^{\prime}$ are normalized by dividing each element by the sum of the entries of its column, the above formulation becomes 


$$
h_{i j}^{\prime}=\frac{\left(\frac{h_{i k}}{v_{i k}}\right) v_{i j}}{\sum_{y=1}^{m}\left(\left(\frac{h_{y k}}{v_{y k}}\right) v_{y j}\right)}
$$

for $i=1,2, \ldots, m$ and $j=1,2, \ldots, n$.

Flexibility in choosing a way to normalized the decision matrix in dual pairwise comparisons is due to the assumption governing it which says "Given matrix $V$ and one row of matrix $H$, then matrix $H$ can be derived according to the above relation, assuming invariance of proportions”.

Triantaphyllou has shown that the number of pairwise comparisons is less in the duality approach than in the original AHP when $m>n+1$, that is $n(n-1) / 2+m n(n-1) / 2+m(m-1) / 2$. Interested readers can refer to Triantaphyllou (1999) for the theorem and corollaries.

\section{A Comparative Study on Time Complexity}

The first issue in the study is the time complexity issue. In the duality approach the number of comparisons is said to be less than the number of comparisons in the original AHP, which at the same time hoped to reduce the amount of time to obtain the overall ranks of the main objective of a problem. The SVD in general, is computable in time $O\left(\min \left\{m n^{2}, m^{2} n\right\}\right)$. A few experiments have been taken to capture the amount of time for both the methods, for different combinations of $n$ and $m(2<n, m<8)$. This is to simulate human's abilities to compare things in pairs in real life.

\subsection{Different Cases of $\boldsymbol{n}$ and $\boldsymbol{m}$}

A function was written in Matlab to obtain the results. For $n=3,100$ random matrices were considered. For $n=4$ and $n=5,80$ random matrices were considered. Below are some of the figures for the cases of different $n$ and $m$.

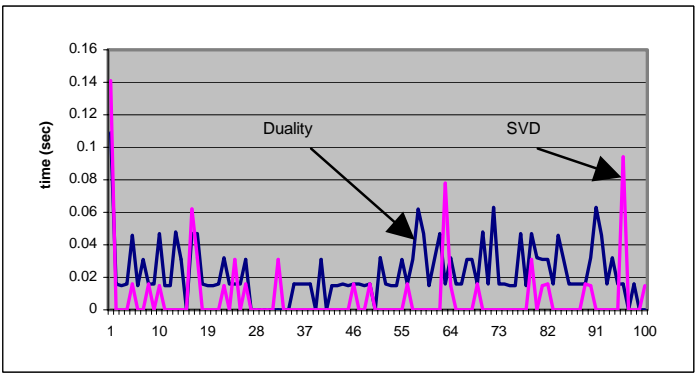

Figure 2: Time comparisons for $n=3$ and $m=5$

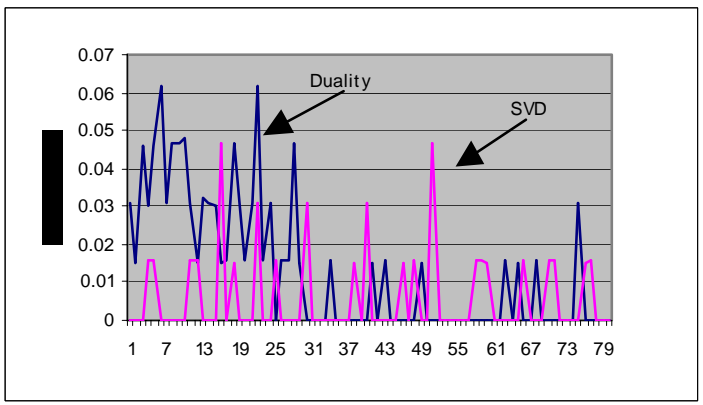

Figure 4: Time comparisons for $n=4$ and $m=6$

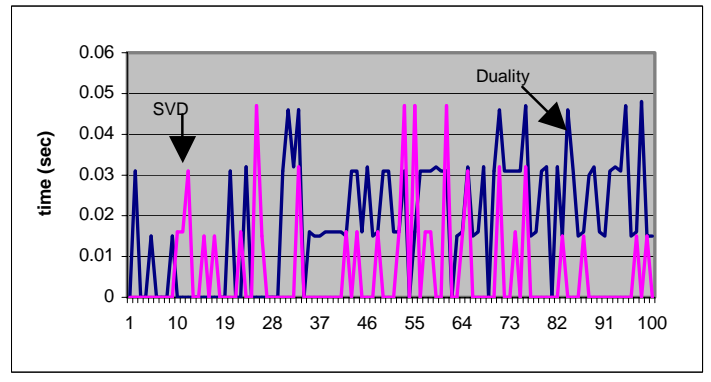

Figure 3: Time comparisons for $n=3$ and $m=6$

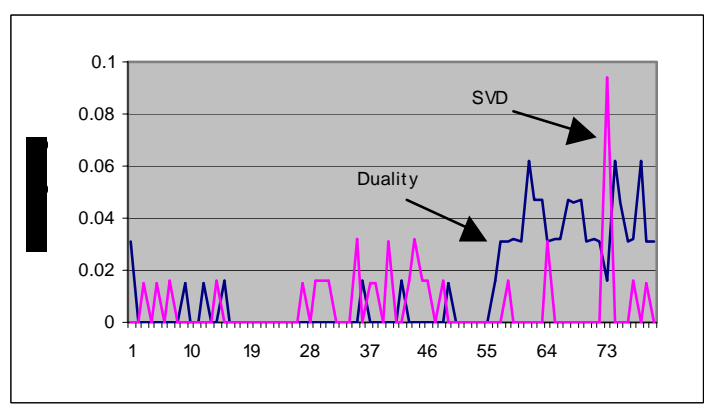

Figure 5: Time comparisons for $n=5$ and $m=7$ 
From the graphs above, it can be seen that the SVD in AHP requires less computational time when compared with the duality approach in AHP.

The study experimented only with cases where $m>n+1$. Defining the time complexity as the time required to obtain the pairwise comparison matrices and later calculate the overall ranking of candidates for the problem, therefore we see that the SVD in AHP is $O\left(m n^{2}\right)$ while the duality approach on the other hand is $O\left(\mathrm{~m}^{3}\right)$.

In addition, the SVD in AHP concentrates on the largest eigenvalue of the pairwise comparison matrix and its corresponding eigenvector only (after the matrix decomposition) which means only the first column of matrices $U$ and $V$ are considered in the calculation. On the other hand, the duality approach even with the reduced number of pairwise comparisons, still searches and calculates through all eigenvalues and after then only pick the largest one.

\section{A Comparative Study on Overall Rank Result}

The second issue in the study is the overall rank result. The sum of the weights of the candidates for both SVD in AHP and duality approach in AHP is one. The issue of concerned here is to see whether both methods give the same overall ranks for the candidates. The objective is to see which method gives a better and more justified result.

All matrices used in this study are the same matrices used in the previous study.

\subsection{Different Cases of $n$ and $m$}

Below is the table from the experiments.

$\begin{array}{lccccccccccc}\underline{\boldsymbol{C .} . \boldsymbol{R}} & \mathbf{0 . 0 0} & \underline{\mathbf{0 . 0 1}} & \underline{\mathbf{0 . 0 2}} & \underline{\mathbf{0 . 0 3}} & \underline{\mathbf{0 . 0 4}} & \underline{\mathbf{0 . 0 5}} & \underline{\mathbf{0 . 0 6}} & \underline{\mathbf{0 . 0 7}} & \underline{\mathbf{0 . 0 8}} & \underline{\mathbf{0 . 0 9}} & \underline{\mathbf{0 . 1}} \\ n=3, m=5 & 100 & 46 & 38 & 40 & 27 & 17 & 50 & 0 & 24 & - & 0 \\ n=3, m=6 & 100 & 17 & 27 & 11 & 0 & 0 & 7 & - & 9 & 0 & 0 \\ n=4, m=6 & 100 & 100 & 57 & 33 & 33 & 0 & 17 & 14 & 17 & 11 & 10 \\ n=5, m=7 & 100 & - & 33 & 20 & 25 & - & 13 & 11 & 0 & 0 & 0\end{array}$

Table 1: Percent (\%) of matches in ranks (different combination of $\boldsymbol{n}$ and $\boldsymbol{m}$ ) for SVD and duality approach in AHP

The critical ratios (C.R.) obtained from the experiments have been rounded to 2 decimal places. From the above table, for all combinations of $n$ and $m$, it can be observed that if the pairwise comparison matrices become less consistent, then the percentage of the number of matches in candidates' rankings tends to reduce. It is also observed that the matrices with nearly perfect consistencies $(0.000<\mathrm{C} . \mathrm{R}<$ 0.001 ) gave the same ranking while the rest resulted in different rankings between the two methods. The results are inline with Gass and Rapcsák (1998) statement that in the case of positive and consistent matrices, the SVD and EM give the same result. Here, although the study does not concern of EM, the duality approach does give the same rank result as the EM when the matrices are of perfect consistencies (Triantaphyllou (1999)).

As $n$ and $m$ get larger, the percent of matrices with the same overall rankings derived from both the SVD and duality approach in AHP tends to reduce. The main reason lies on the difficulties of obtaining consistent matrices (C.R. < 0.1).

The SVD together with the theory of low rank approximation of a pairwise comparison matrix (Theorem 2.1.2) have been used by Gass and Rapcsák (1998) to prove theoretically that the rank one left and right singular vectors (from the matrix decomposition), or the vectors that are associated with the largest 
singular value, yield justified weights/ranks. The duality approach in AHP on the other hand, was designed and proposed to reduce the number of pairwise comparisons especially when $m>n+1$. The ranks of the dual matrices are smaller when compared to the original matrices in AHP, but are still larger than the rank one of the SVD matrices.

\section{Conclusion}

Our experiment showed that the SVD in AHP gave a better advantage to the decision maker in selecting the best candidate to be in a faculty when compared to the duality approach in AHP. The amount of time required to obtain the overall ranks is less when using the SVD because the number of pairwise comparisons is less and the SVD concentrates only on the one largest eigenvalue and its corresponding eigenvector, saving the decision maker's limited time. In terms of the acceptability of the decisions, the SVD produces more justified weights. In the real application of selecting the best candidate for a faculty, with the number of candidates larger than the number of selection criteria, the SVD in AHP is suggested for it's time saving property and the more justified result.

\section{References}

Badri, M. (2001) “Combining the AHP and GP Model for Quality Control Systems”, International Journal of Production Economics 72, 27-40

Belton, V. and Gear, T. (1983) “On a Short-coming of Saaty’s Method of Analytic Hierarchies”, Omega, 228-230.

Boender, C.G.E., de Graan, J.G. and Lootsma, F.A. (1989) "Multi-Criteria Decision Analysis with Fuzzy Pairwise Comparisons”, Fuzzy Sets and Systems, Vol.29, 133-143

Donegan, H.A., Dodd, F.J. and McMaster, T.B.M. (1992) “A New Approach to AHP Decision-making”, The Statistician, 41, 295-302.

Dyer, J.S. and Wendell, R.E. (1985) “Irrelevant Alternatives and the Analytic Hierarchy Process”, Technical Report, Department of Management, The University of Texas at Austin, Austin, TX

Eckart,C. and Young, G. (1936) “The Approximation of One Matrix by Another of Lower Rank”, Pscychometrika, 1, 211-218

Escobar M. T. and Moreno-Jiménez J. M. (2000) "Reciprocal Distributions in the Analytic Hierarchy Process”, European Journal of Operational Research, 123, 154-174

Gass, S.I. and Rapcsák, T. (2004) “Singular Value Decomposition in AHP”, European Journal of Operational Research, 154,573-584

Gass, S.I. and Rapcsák, T. (1998) “A Note on Synthesizing group decisions”, Decision Support Systems, 22, 59-63

Ghodsypur, S.H. and O’Brien, C. (1998) “A Decision Support System for Supplier Selecting Using an Integrated Analytic Hierarchy Process and Linear Programming”, International Journal of Production Economics, 56-57, 199-212

Greenacre, M.J. (1984) Theory and Applications of Corresponding Analysis, Academic Press, London, Orlando

Iwamura, K. and Lin, B. (1998) “Chance Constrained Integer Programming Models for Capital Budgeting Environments”, Journal of Operational Research, 116, 423-435. 
Johnson C.R., Beine W.B. and Wang T.J. (1979) "Right-left Asymmetry in an Eigenvector Ranking Procedure”, Journal of Mathematical Psychology, 19, 61-64

Laarhoven, P.J.M. and Pedrycz, W. (1983) “A Fuzzy Extension of Saaty’s Priority Theory”, Fuzzy Sets and Systems, Vol. 11, 229-241

Labib, A.W., Williams, G.B. and O’Connor, R.F. (1998) “An Intelligent Maintenance Model (System): An Application of the Analytic Hierarchy Process And A Fuzzy Rule-based Controller”, Journal of Operational Research Society 49, 745-757.

Lai, Y.J. (1995) “IMOST: Interactive Multiple Objective System Technique”. Journal of Operational Research Society 46, 958-976.

Petrovic-Lazarevic, S. (2001) “Personnel Selection Fuzzy Model”, International Transactions in Operational Research 8, 89-105.

Radasch, D.K. and Kwak, N.K. (1998), “An Integrated Mathematical Programming Model for Offset Planning”, Computers and Operations Research 25, 1069-1083

Ramanathan, R. and Ganesh, S.H. (1995) “Using AHP for Resource Allocation Problems”, European Journal of Operational Research 80, 410-417

Saaty, T.L. (1980) The Analytic Hierarchy Process, McGraw-Hill, New York

Saaty, T.L. (1994) Fundamentals of Decision Making and Priority Theory with the AHP, RWS Publications, Pittsburgh, PA, USA

Triantaphyllou, E. (1999) "Reduction of Pairwise Comparisons in Decision Making via a Duality Approach”, Journal of Multi-Criteria Decision Analysis, 8, 299-310

Triantaphyllou, E. and Mann, S.H. (1994) "Some Critical Issues in Making Decisions with Pairwise Comparisons", Proceedings of the Third International Symposium on the Analytic Hierarchy Process, Washington, DC, 225-235

Yager, R.R (1978) “Fuzzy Decision Making Using Unequal Objectives”, Fuzzy Sets and Systems, 1, 8795. 